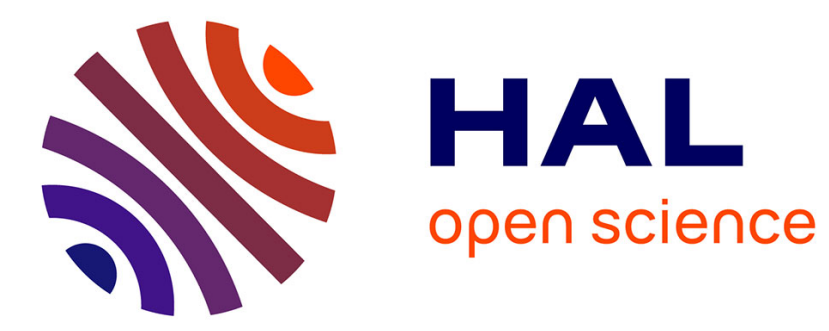

\title{
Étude par couplage inductif du facteur de surtension d'échantillons conducteurs résonnants
}

\author{
J. Mazuer, Y. Brunet, J. Gilchrist, P. Monceau, J. Odin
}

\section{To cite this version:}

J. Mazuer, Y. Brunet, J. Gilchrist, P. Monceau, J. Odin. Étude par couplage inductif du facteur de surtension d'échantillons conducteurs résonnants. Revue de Physique Appliquée, 1971, 6 (3), pp.377381. 10.1051/rphysap:0197100603037700 . jpa-00243559

\section{HAL Id: jpa-00243559 https://hal.science/jpa-00243559}

Submitted on 1 Jan 1971

HAL is a multi-disciplinary open access archive for the deposit and dissemination of scientific research documents, whether they are published or not. The documents may come from teaching and research institutions in France or abroad, or from public or private research centers.
L'archive ouverte pluridisciplinaire HAL, est destinée au dépôt et à la diffusion de documents scientifiques de niveau recherche, publiés ou non, émanant des établissements d'enseignement et de recherche français ou étrangers, des laboratoires publics ou privés. 


\title{
ÉTUDE PAR COUPLAGE INDUCTIF DU FACTEUR DE SURTENSION D'ÉCHANTILLONS CONDUCTEURS RÉSONNANTS
}

\author{
J. MAZUER (*), Y. BRUNET, J. GILCHRIST, P. MONCEAU et J. ODIN (*) \\ Centre de Recherches sur les Très Basses Températures, C. N. R. S., Cedex 166 \\ 38, Grenoble-Gare
}

(Reçu le ler $^{\text {avril 1971) }}$

\begin{abstract}
Résumé. - Nous décrivons deux montages qui permettent de déterminer le facteur de surtension d'échantillons conducteurs en résonance électromagnétique à basse température, l'un à $100 \mathrm{MHz}$, l'autre à $2,4 \mathrm{GHz}$. Un même circuit équivalent, à composants discrets, sert à analyser les résultats obtenus avec les deux montages. Nous montrons l'application de ces montages à l'étude d'une ligne coaxiale supraconductrice de $1,42 \mathrm{~m}$ de long, d'une part, et à la détermination des propriétés de supraconducteurs de type II dans un champ magnétique, d'autre part. L'examen des résonances nous permet d'affirmer la validité du circuit et le bon fonctionnement des appareils.
\end{abstract}

\begin{abstract}
We describe two experimental setups with which we can determine the Q-factor of conducting specimens in electromagnetic resonance at low temperature, one at $100 \mathrm{MHz}$, the other at $2.4 \mathrm{GHz}$. The same equivalent circuit of lumped components is adopted for the analysis of the results from each setup. We show the applications to the study of a $1.42 \mathrm{~m}$ long superconducting coaxial line and to the determination of the properties of type II superconductors in a magnetic field. By examining the resonances we can affirm that the equivalent circuit is valid and that the two circuits function correctly.
\end{abstract}

I. Introduction. - Il existe plusieurs méthodes pour déterminer les caractéristiques électriques à haute fréquence d'échantillons métalliques. Les trois principales sont la méthode calorimétrique, la mesure de l'inductance d'une bobine entourant l'échantillon et la méthode de la résonance. Toutes les trois ont été employées pour étudier les supraconducteurs [1], [2], [3].

La méthode de la résonance permet d'accéder par la mesure du facteur de surtension à la résistance de surface de l'échantillon. Pour les fréquences supérieures à $3 \mathrm{GHz}$ on utilise des cavités résonnantes et des guides d'ondes. Pour les fréquences plus faibles l'échantillon résonnant peut prendre plusieurs formes. Ainsi, Pippard [3] a utilisé un résonateur quart d'onde en épingle à cheveux et Waldram [4] un échantillon comme conducteur central d'une ligne coaxiale demi-onde. Le circuit est alors formé de lignes de transmission et le couplage entre échantillon et circuit peut se faire soit par induction, soit par capacité. Le couplage inductif est le plus communément utilisé, et se présente sous deux formes. L'échantillon peut être couplé magnétiquement à deux antennes en forme de boucles. Il faut alors minimiser le couplage direct entre l'antenne de transmission et l'antenne de réception. L'analyse d'un tel circuit a été faite par Waldram [4]. L'autre solution

(*) Laboratoire d'Electrotechnique, Institut National Polytechnique, 46 , avenue F. Viallet, 38 , Grenoble. consiste à avoir une seule boucle qui relie la ligne d'entrée à la ligne de sortie. La résonance de l'échantillon apparaît, dans ce cas, comme une résonance d'absorption, à condition de compenser l'inductance de la boucle de façon à permettre la transmission intégrale de la puissance en l'absence de résonance. C'est ce dernier système que nous décrivons et analysons dans les paragraphes suivants.

II. Les deux montages expérimentaux. - Le 'montage pour $100 \mathrm{MHz}$ est illustré, en schéma, par la figure 1. Il comprend trois parties : a) le circuit haute fréquence, $b$ ) un circuit basse fréquence et $c$ ) un circuit à courant continu.

a) Le signal haute fréquence modulé en amplitude est fourni par un générateur VHF Férisol type L $201 \mathrm{~A}$. Deux détecteurs à diode (Hewlett-Packard, type $423 \mathrm{~A}$ ) servent à déterminer le niveau de puissance à l'entrée et à la sortie du cryostat. Nous nous assurons que les deux détecteurs fonctionnent dans la zone quadratique de leur caractéristique tension de sortie-tension d'entrée. Pour cela la tension d'entrée reste toujours inférieure à $50 \mathrm{mV}$. L'échantillon résonnant à étudier est installé dans un cryostat de $2,20 \mathrm{~m}$ de hauteur. Sur la figure 1 nous l'avons représenté comme constitué de deux fils parallèles, court-circuités aux deux extrémités. Il peut également être ouvert à l'extrémité inférieure, ou encore être de type coaxial. L'inductance de la boucle de couplage est compensée symboliquement par 


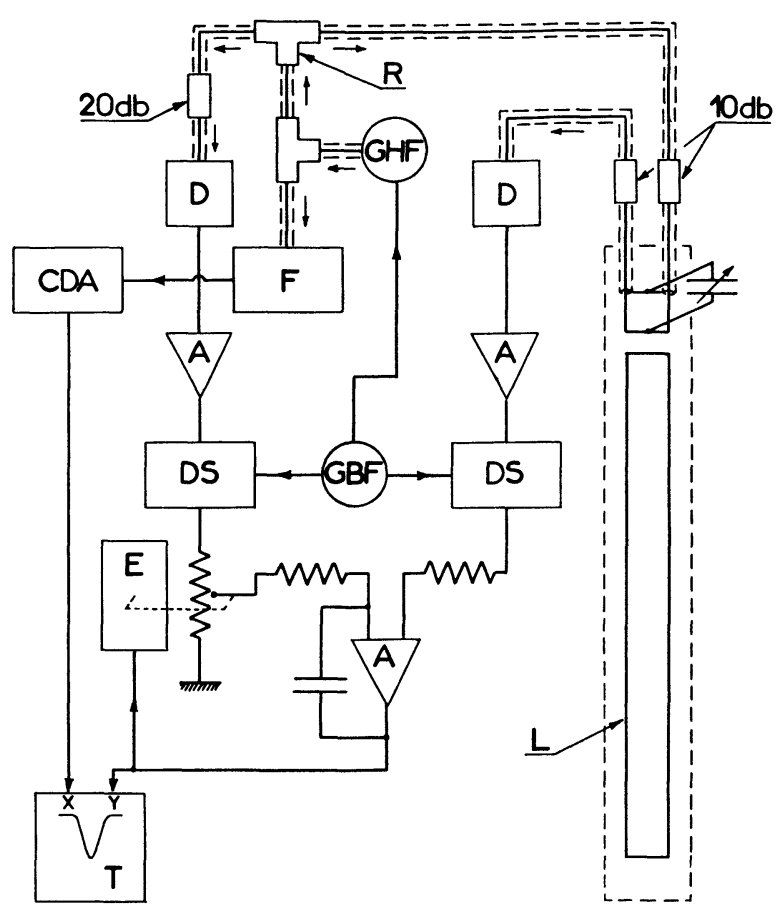

FIG. 1. - Schéma du montage pour $100 \mathrm{MHz}$ : la partie hautefréquence du schéma (en haut et à droite) est délimitée par les liaisons blindées qui représentent des câbles $50 \Omega$; A, amplificateur ; CDA, convertisseur digital-analogique ; $\mathrm{D}$, détecteur ; DS, détecteur synchrone ; E, enregistreur potentiométrique lié à un potentiomètre de répétition; $F$, fréquencemètre ; $G B F$, générateur $400 \mathrm{~Hz}$; GHF, générateur haute fréquence modulé par $\mathrm{GBF} ; \mathrm{L}$, ligne résonnante à étudier $; \mathrm{R}$, répartiteur de puissance ; $\mathrm{T}$, table traçante; les atténuateurs fixes sont repérés par leur coefficient d'atténuation.

une capacité variable, mais dans la réalité nous avons trouvé que la capacité parasite seule suffisait.

b) Le circuit basse fréquence est constitué d'un générateur $400 \mathrm{~Hz}$, de deux amplificateurs bas niveau et de deux détecteurs synchrones. Le générateur bassefréquence fournit le signal de modulation du générateur haute-fréquence et la référence des détecteurs synchrones.

c) Un servomécanisme de position à courant continu, comprenant un amplificateur différentiel, un enregistreur potentiométrique et un potentiomètre de répétition effectue le quotient des tensions de sortie des deux détecteurs synchrones. Ce quotient est directement proportionnel au rapport des niveaux de puissance dans le circuit haute-fréquence à l'entrée et à la sortie du cryostat et aussi au coefficient de transmission en puissance, $T$, de la boucle de couplage. Le quotient est obtenu avec une bonne précision grâce à la valeur du gain de l'amplificateur différentiel $\left(\simeq 3 \times 10^{4}\right)$. La tension de sortie de cet amplificateur est envoyée sur l'entrée $Y$ d'une table traçante dont l'entrée $X$ est alimentée par un signal qui varie linéairement avec la fréquence du générateur GHF, signal obtenu grâce à l'ensemble fréquencemètre-convertisseur digital-analogique. Ce dispositif permet ainsi de tracer directement les courbes de $T$ en fonction de la fréquence.
Le montage pour $2,4 \mathrm{GHz}$ a été décrit dans une publication antérieure [5]. Il est très similaire au précédent, mais il y a plusieurs différences de détail à signaler. Ainsi, pour compenser l'inductance de la boucle nous avons dû prévoir un élément réactif constitué par une courte ligne de transmission dans laquelle peut coulisser un tube de quartz. La position du quartz est réglée de l'extérieur du cryostat. Le signal haute-fréquence n'est pas modulé, et les deux amplificateurs bas niveau suivis des détecteurs synchrones sont remplacés par deux amplificateurs du type galvanométrique à courant continu. D'autre part, il n'a pas été utilisé de convertisseur digital analogique ni de table traçante. Signalons finalement que pour les deux montages le couplage entre boucle et échantillon peut être modifié de l'extérieur du cryostat par un dispositif mécanique.

III. Circuit équivalent. - Le résonateur peut être assimilé à un circuit résonnant série. Soit $\mathrm{R}_{3}, \mathrm{C}_{3}, \mathrm{~L}_{3}$ ses caractéristiques. Si les deux parties de la boucle de couplage ont des inductances $L_{1}$ et $L_{2}$, et une inductance mutuelle $M_{12}$, l'inductance $L_{\mathrm{b}}$, de toute la boucle est $\left(L_{1}+L_{2}+2 M_{12}\right) . L_{1}$ est reliée au générateur d'impédance $R_{1}$ par une ligne adaptée. Le générateur et la ligne peuvent être remplacés par une source équivalente constituée d'une force électromotrice $E \mathrm{e}^{i \omega t}$ et d'une résistance $R_{1}$ en série. De la même manière, on peut imaginer que $L_{2}$ est branché à une simple charge $R_{2}$ dans laquelle nous mesurons la dissipation $\frac{1}{2} V^{2} R_{2}^{-1}$. La figure 2 donne le schéma du circuit ainsi décomposé. Remarquons que nous avons supposé que les lignes de transmission et les autres composants sont parfaitement accordés en impédance. Le rôle des atténuateurs est précisément de diminuer l'effet néfaste des mauvais accords.

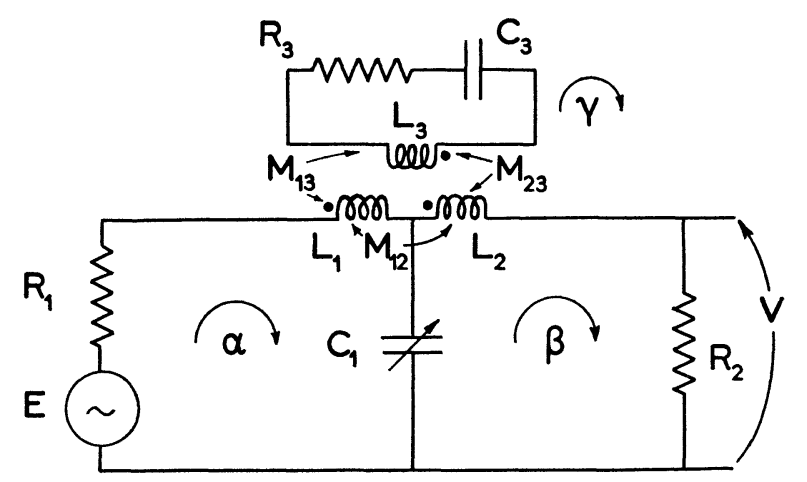

FIG. 2. - Circuit haute-fréquence équivalent.

ANALYSE DU CIRCUIT ÉQuivalent. - Considérons le montage sans résonateur et la boucle exactement compensée par la capacité d'accord $C_{1}$. Puisque $R_{2}=R_{1}$, nous voyons que $|V|=\frac{1}{2} E$. Alors en présence du résonateur, $T=|t|^{2}$, où $t$, le coefficient de transmission en tension s'exprime par $2 V E^{-1}$ ou $2 R_{1} \beta E^{-1}$. 
La méthode des courants fictifs de Maxwell nous donne pour le circuit de la figure 2 :

$$
\begin{gathered}
E=\left[R_{1}+j\left(\omega L_{1}-\omega^{-1} C_{1}^{-1}\right)\right] \alpha+ \\
\quad+j\left(\omega M_{12}+\omega^{-1} C_{1}^{-1}\right) \beta+j \omega M_{13} \gamma, \\
0=j\left(\omega M_{12}+\omega^{-1} C_{1}^{-1}\right) \alpha+ \\
+\left[R_{2}+j\left(\omega L_{2}-\omega^{-1} C_{1}^{-1}\right)\right] \beta+j \omega M_{23} \gamma, \\
0=j \omega M_{13} \alpha+j \omega M_{23} \beta+ \\
\quad+\left[R_{3}+j\left(\omega L_{3}-\omega^{-1} C_{3}^{-1}\right)\right] \gamma .
\end{gathered}
$$

Nous nous bornons au cas où le circuit est bien symétrique, c'est-à-dire $L_{2}=L_{1}, R_{2}=R_{1}$ et $M_{23}=M_{13}$. Posons

$$
\left(L_{3} C_{3}\right)^{1 / 2}=\omega_{0}, \quad R_{3}^{-1} L_{3}^{1 / 2} C_{3}^{-1 / 2}=Q
$$

et

$$
\omega=\omega_{0}\left(1+\frac{1}{2} Q^{-1} \delta\right) .
$$

Tant que $\omega \approx \omega_{0},(1 c)$ devient :

$$
\gamma \approx-j Q M_{13} L_{3}^{-1}(1+j \delta)^{-1}(\alpha+\beta) .
$$

La somme des équations $(1 a)$ et $(1 b)$ donne une relation entre $E,(\alpha+\beta)$ et $\gamma$, et leur différence relie $E$, $(\alpha-\beta)$ et $\gamma$. En écrivant $t$ sous la forme

$$
\left[R_{1}(\alpha+\beta) E^{-1}-R_{1}(\alpha-\beta) E^{-1}\right]
$$

il vient :

$$
\begin{aligned}
& t \approx\left[1+j \omega_{0}\left(L_{1}+M_{12}\right) R_{1}^{-1}+A_{0} Q(1+j \delta)^{-1}\right]^{-1}- \\
& -\left[1+j \omega_{0}\left(L_{1}-M_{12}\right) R_{1}^{-1}-2 j\left(\omega_{0} C_{1} R_{1}\right)^{-1}\right]^{-1} .
\end{aligned}
$$

Dans cette expression le couplage entre le résonateur et le reste du circuit s'exprime par le paramètre $A_{0}$ :

$$
A_{0}=2 \omega_{0} M_{13}^{2} L_{3}^{-1} R_{1}^{-1} \text {. }
$$

Il suffit d'imposer la condition $|t|=1$ quand $A_{0}=0$ pour trouver que la bonne valeur de $C_{1}$ est :

$$
L_{\mathrm{b}}\left[R_{1}^{2}+\omega_{0}^{2}\left(L_{1}^{2}-M_{12}^{2}\right)\right]^{-1} .
$$

Avec cette valeur de $C_{1}$, (2) devient :

$$
\begin{aligned}
t \approx(1+j \delta)[(1+j s)(1+j \delta) & \left.+A_{0} Q\right]^{-1}- \\
& -\left(1-j s^{-1}\right)^{-1},
\end{aligned}
$$

où

$$
s=\frac{1}{2} \omega_{0} L_{\mathrm{b}} R_{1}^{-1} .
$$

Introduisons encore un symbole :

$$
A=A_{0}\left(1+s^{2}\right)^{-1},
$$

pour exprimer le coefficient de transmission en puissance :

$$
\begin{aligned}
T=1- & {\left[(1+A Q)^{2}-1\right] \times } \\
\times & {\left[(\delta-s A Q)^{2}+(1+A Q)^{2}\right]^{-1} . }
\end{aligned}
$$

Rappelons que dans cette expression, les quatre symboles représentent des quantités adimensionnelles. $s$ caractérise la boucle de couplage, $A$ exprime l'intensité de couplage, $Q$ est la propriété du résonateur que nous voulons connaître et $\delta$ définit la fréquence. Lorsque $\delta=s A Q, T(\omega)$ atteint sa valeur minimale :

$$
T_{\mathrm{m}}=(1+A Q)^{-2} .
$$

$T$ peut s'exprimer à l'aide de $T_{\mathrm{m}}$ :

$$
T=1-\left(1-T_{\mathrm{m}}\right)\left[1+\dot{T}_{\mathrm{m}}(\delta-s A Q)^{2}\right]^{-1} .
$$

DÉTERMINATION DU FACTEUR DE SURTENSION. Nous remarquons d'abord que par la seule mesure de $T_{\mathrm{m}}$ nous pouvons déterminer la variation de $Q$ en fonction d'un quelconque paramètre, $P$ (par exemple la température). L'inversion de l'expression (5) donne

$$
A Q=\left(T_{\mathrm{m}}^{-1 / 2}-1\right) \text {. }
$$

En général, nous ignorons la valeur précise de $A$ mais nous pouvons nous assurer qu'elle reste constante pendant la durée d'une manipulation. Alors

$$
\frac{Q\left(P_{1}\right)}{Q\left(P_{0}\right)}=\frac{T_{\mathrm{m}}^{-1 / 2}\left(P_{1}\right)-1}{T_{\mathrm{m}}^{-1 / 2}\left(P_{0}\right)-1},
$$

où $P_{0}, P_{1}$ sont deux valeurs de $P$. Ce mode de travail permet des mesures rapides et simples. L'étalonnage en fréquence de la courbe n'est pas essentiel. Dans le paragraphe $\mathrm{V}$ nous verrons une application où il importe peu de connaître la valeur absolue des facteurs de surtension.

Nous pouvons ensuite exploiter la largeur des résonances, c'est-à-dire l'intervalle de fréquence entre les deux points où $T=\frac{1}{2}\left(1+T_{\mathrm{m}}\right)$. Appelons cette intervalle $\Delta$. De l'expression (4 bis) nous voyons que

$$
T=\frac{1}{2}\left(1+T_{m}\right)
$$

lorsque

$$
\delta=s A Q \pm T_{\mathrm{m}}^{-1 / 2}
$$

D'où

$$
\Delta=\omega_{0} Q^{-1} T_{\mathrm{m}}^{-1 / 2},
$$

et

$$
Q^{(1)}=\omega_{0} \Delta^{-1} T_{\mathrm{m}}^{-1 / 2} .
$$

La détermination de $Q$ nécessite, alors, de chiffrer $\Delta$ et $T_{\mathrm{m}}$. Cependant il existe une autre méthode qui est plus directe et qui consiste à trouver la largeur à mihauteur de la courbe $T^{-1}$, c'est-à-dire l'intervalle $\Delta_{0}$ entre points où

$$
T^{-1}=\frac{1}{2}\left(1+T_{\mathrm{m}}^{-1}\right) .
$$

A partir de (4 bis), $Q$ est donné par

$$
Q^{(2)}=\omega_{0} \Delta_{0}^{-1} .
$$

Sur la figure 3 nous avons tracé trois courbes de résonance à partir de la relation (4 bis). Les valeurs des 
différents paramètres sont déduites des points expérimentaux. Sur une des courbes nous avons fait apparaître les largeurs $\Delta$ et $\Delta_{0}$.

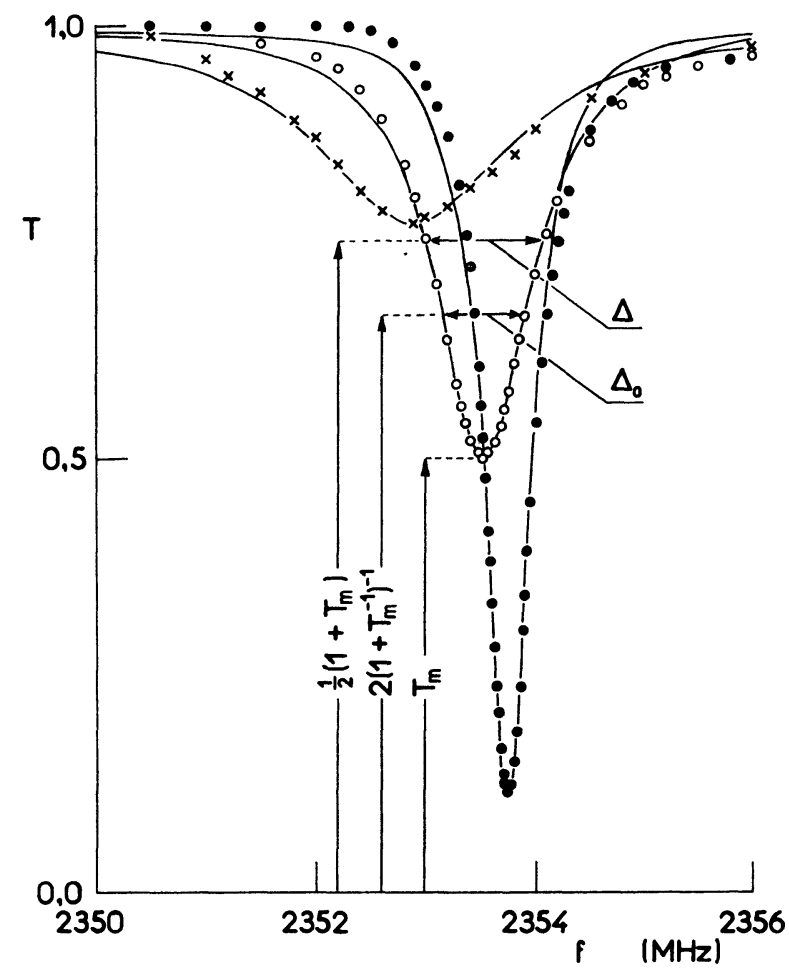

Fig. 3. - Résonances d'un échantillon $\mathrm{Pb}_{50} \mathrm{In}_{50}$ à $4,2^{\circ} \mathrm{K}$, en champ nul (0), $\mu_{0} H_{0}=0,095$ Teslas (o), et 0,5 T $(\times)$. Dans ces trois conditions l'échantillon est respectivement à l'état Meissner, l'état mixte et l'état normal. Les courbes correspondent à l'expression $(4 \mathrm{bis})$ avec les paramètres choisis convenablement.

Nous avons remarqué, au moment d'écrire (4 bis) que la résonance est symétrique autour d'une fréquence définie par $\delta-s A Q=0$, que nous appellerons $(2 \pi)^{-1} \omega_{r} . \omega_{r}$ diffère de $\omega_{0}$ par un facteur $\left(1+\frac{1}{2} s A\right)$. L'écart entre $\omega_{r}$ et $\omega_{0}$ est donc proportionnel au paramètre de couplage, et aussi à $L_{\mathrm{b}}$. Pour situer l'importance de l'écart nous pouvons remarquer que

$$
\left(\omega_{r}-\omega_{0}\right) \Delta_{0}^{-1}=\frac{1}{2} s A Q=\frac{1}{2} s\left(T_{\mathrm{m}}^{-1 / 2}-1\right) .
$$

Afin d'éviter un trop grand écart qui pourrait être nuisible aux mesures, il est souhaitable que $s \lesssim 1$. Pour nos deux montages, $s=0,25$ (100 MHz), $s=2,3$ $(2,4 \mathrm{GHz})$.

IV. Ligne supraconductrice à 100 MHz. - La ligne coaxiale étudiée est constituée d'un fil de niobium de diamètre $0,1 \mathrm{~mm}$ et d'un tube de cuivre de diamètre 20-22 $\mathrm{mm}$ recouvert intérieurement d'une couche de plomb-étain. La ligne, de 1,42 $\mathrm{m}$ de long, fermée à chaque extrémité, est plongée dans l'hélium liquide à $4,2 \mathrm{~K}$. Elle résonne en demi-onde à $102,1 \mathrm{MHz}$. La boucle de couplage entre dans la ligne par une fente latérale située près d'une extrémité. Nous présentons ici les résultats d'une étude de la variation du coefficient de surtension en fonction du couplage bouclerésonateur. Pour chaque courbe de résonance nous avons déterminé $\omega_{r} \Delta^{-1}, Q^{(1)}$ et $Q^{(2)}$ (voir expressions [6] et [7]). Sur la figure 4 les valeurs de ces trois quantités sont représentées en fonction de $T_{\mathrm{m}}$.

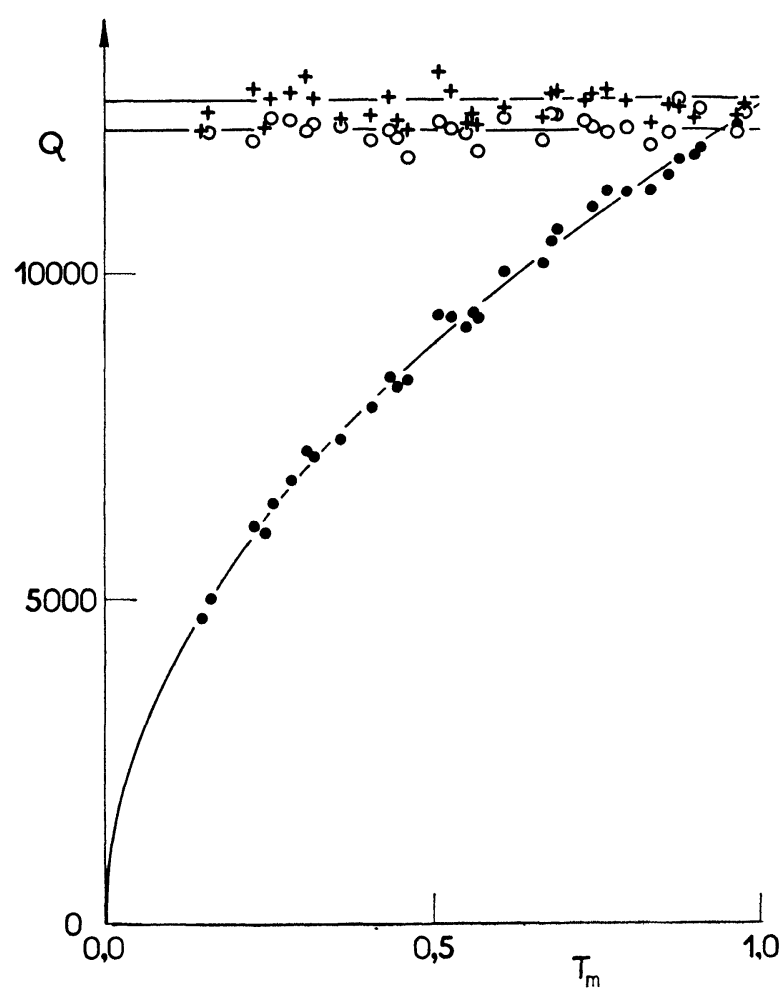

Fig. 4. $-\omega_{r} \Delta^{-1}(\bullet), Q^{(1)}(+)$ et $Q^{(2)}(0)$ pour la ligne résonnante à $100 \mathrm{MHz}$, avec différents couplages. Les points qui correspondent à $T_{\mathrm{m}} \ll 1$ sont obtenus avec le couplage le plus fort, et ceux qui correspondent à $T_{\mathrm{m}} \approx 1$ avec le couplage le plus faible.

L'examen de la figure 4 appelle plusieurs remarques. Les points qui représentent $Q^{(1)}$ et $Q^{(2)}$ semblent être dispersés de façon aléatoire autour des valeurs moyennes 12700 et 12200 . $Q^{(1)}$ et $Q^{(2)}$ sont indépendants du couplage, comme nous l'attendions, mais la différence constatée entre les deux valeurs moyennes peut s'expliquer par un écart de la courbe expérimentale de résonance à la forme lorentzienne. $Q^{(2)}$, calculé à partir de la courbe inverse de celle enregistrée est cependant la valeur dans laquelle nous mettons le plus de confiance, puisqu'elle fait intervenir une bande de fréquences plus étroite. L'extrapolation du rapport $\omega_{r} \Delta^{-1}$ à couplage nul $\left(T_{\mathrm{m}} \rightarrow 1\right)$ fournit une autre valeur de $Q$, voisine de $Q^{(1)}$, mais c'est un processus laborieux qui présente peu d'intérêt.

Il faut noter que pendant ces mesures les pertes sont d'environ $10 \mu \mathrm{W}$ pour une énergie électromagnétique stockée voisine de $100 \mathrm{pJ}$. Le champ haute fréquence maximal à la superficie du fil est de l'ordre de $50 \mathrm{Am}^{-1}$.

V. Supraconducteur de type II à $\mathbf{2 , 4} \mathbf{~ G H z}$. - Sur la figure 3 nous présentons les courbes de résonance d'un échantillon de supraconducteur de type II pour trois valeurs du champ magnétique statique, $H_{0}$, appliqué perpendiculairement à l'échantillon. Plus précisément, 
les points représentent les résultats expérimentaux et les courbes représentent la fonction $(4 \mathrm{bis})$. Nous observons un léger décalage entre les valeurs mesurées et les courbes. Cet écart peut être dû à un mauvais accord des éléments du circuit haute fréquence. La correspondance entre points et courbes est cependant assez satisfaisante.

$\mathrm{Q}$ est inversement proportionnel à la somme des pertes du résonateur et peut s'écrire :

$$
Q^{-1}\left(H_{0}\right)=Q_{0}^{-1}+\alpha R\left(H_{0}\right),
$$

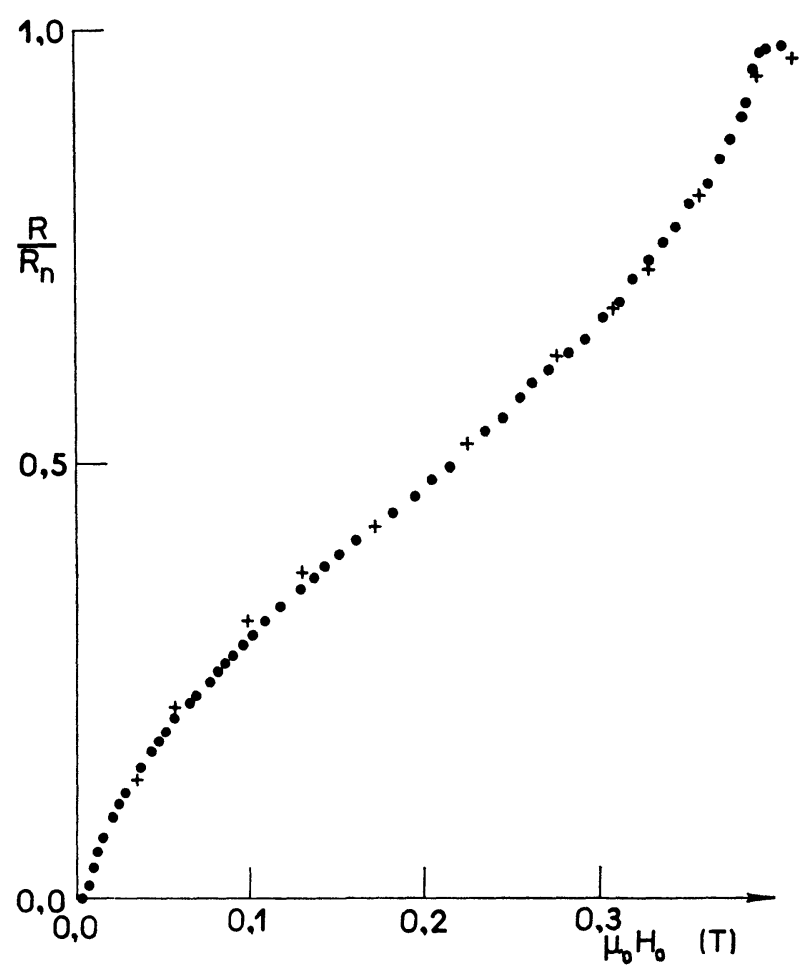

Fig. 5. - Rapport entre la résistance de surface de l'échantillon $\mathrm{Pb}_{50} \mathrm{In}_{50}$ à $4,2^{\circ} \mathrm{K}$ en champ $H_{0}$, et la résistance de surface à l'état normal : déterminé à partir de $T_{\mathrm{m}}(\bullet)$ et à partir de $\Delta(+)$.

où $\alpha$ est une constante et $R\left(H_{0}\right)$ est la résistance de surface du supraconducteur, tandis que $Q_{0}$ est déterminé par les pertes extérieures au supraconducteur (pertes diélectriques, par rayonnement, etc.). Nous supposons que ces pertes extérieures sont indépendantes de $H_{0}$. Il est connu que $R(0)$ est de plusieurs ordres de grandeur plus faible que $\boldsymbol{R}_{\boldsymbol{n}}$ (l'indice $n$ signifie l'état normal avec $\mu_{0} H_{0}>0,5$ Teslas). Or, la valeur $Q(0)$ de la surtension mesurée en champ nul est seulement 8 fois plus forte que $Q_{n}$, la valeur pour l'état normal. Il faut conclure que le terme $Q_{0}^{-1}$ est alors dominant et que $Q(0) \simeq Q_{0}$. Il vient :

$$
\frac{R\left(H_{0}\right)}{R_{n}}=\frac{Q^{-1}\left(H_{0}\right)-Q^{-1}(0)}{Q_{n}^{-1}-Q^{-1}(0)} .
$$

A l'aide de l'expression (5), ce rapport peut s'écrire :

$$
\begin{aligned}
\frac{R\left(H_{0}\right)}{R_{n}} & =\left[\left(T_{\mathrm{m}}^{-1 / 2}\left(H_{0}\right)-1\right)^{-1}-\left(T_{\mathrm{m}}^{-1 / 2}(0)-1\right)^{-1}\right] \times \\
& \times\left[\left(T_{\mathrm{m} n}^{-1 / 2}-1\right)^{-1}-\left(T_{\mathrm{m}}^{-1 / 2}(0)-1\right)^{-1}\right]^{-1} .
\end{aligned}
$$

En plus, il convient de rappeler que $\Delta=\omega_{0}\left(A+Q^{-1}\right)$, d'où :

$$
\frac{R\left(H_{0}\right)}{R_{n}}=\frac{\Delta\left(H_{0}\right)-\Delta(0)}{\Delta_{n}-\Delta(0)} .
$$

Sur la figure 5, nous avons représenté la fonction $R\left(H_{0}\right) / R_{n}$ pour notre échantillon, déterminée par ces deux méthodes. Il y a un excellent accord entre les deux séries de points expérimentaux, ce qui semble signifier que les deux méthodes sont parfaitement valables.

Dans cette étude l'ordre de grandeur des pertes mises en évidence est de $10 \mu \mathrm{W}$. Le champ magnétique maximal haute fréquence qui règne au voisinage de la surface des échantillons est environ de $1 \mathrm{Am}^{-1}$.

Conclusion. - Les deux montages décrits permettent d'étudier les facteurs de surtension dans deux zones de fréquence qui diffèrent de plus d'un ordre de grandeur. Un même circuit équivalent sert à analyser les deux montages. Les résultats que nous avons présentés se rapportent d'une part à une étude en fonction du couplage, à facteur de surtension constant, et d'autre part à une étude de variation du facteur de surtension à couplage constant. La méthode de mesure conduit à une précision de l'ordre de $2 \%$ dans la détermination de la valeur absolue de $Q$ mais la précision est bien meilleure lorsqu'on étudie les variations de cette valeur. Les $Q$ que nous mesurons sont de l'ordre de $10^{4}$, mais la méthode peut être utilisée pour des $Q$ compris entre $10^{3}$ et une limite supérieure déterminée par la stabilité en fréquence du générateur. C'est à partir d'un facteur de surtension de l'ordre de $10^{7}$ qu'il faut utiliser d'autres techniques, par exemple la méthode du décrément.

Cette étude a pu être menée à bien grâce à la collaboration technique de $\mathrm{M}$. Tomasik.

\section{Bibliographie}

[1] Fawcett (E.), Proc. Roy. Soc. A., 1955, 232, 519.

[2] Schawlow (A. L.) et Devlin (G.E.), Phys. Rev., 1959, 113, 120.

[3] Pippard (A. B.), Proc. Roy. Soc. A., 1947, 191, 370.
[4] Waldram (J. R.), Advances in Physics, 1964, 13, 1. [5] Gilchrist (J.), et Monceau (P.), J. Phys. C. Solid St. Phys., 1970, 3, 1399. 